\title{
Lipshitz Continuity of Gap Boundaries for Hofstadter-like Spectra
}

\author{
J. Bellissard ${ }^{\star}, \star \star$ \\ Laboratoire Physique Quantique, Université Paul Sabatier, F-31062 Toulouse Cedex, France
}

Received: 11 March 1993/in revised form: 13 May 1993

\begin{abstract}
We consider an effective Hamiltonian $H$ representing the motion of a single-band-two-dimensional electron in a uniform magnetic field. Then $H$ belongs to the rotation algebra, namely the algebra of continuous functions over a noncommutative 2-torus. We define a non-commutative analog of smooth functions by mean of elements of class $C^{l, n}$, where $l$ and $n$ characterize respectively the degree of differentiability with respect to the magnetic field and the torus variables. We show that if $H$ is of class $C^{1,3+\varepsilon}$, the gap boundaries of the spectrum of $H$ are Lipshitz continuous functions of the magnetic field at each point for which the gap is open.
\end{abstract}

\section{Introduction}

The motion of a single-band-two-dimensional electron in a uniform magnetic field can be represented by an effective Hamiltonian $H$ in the tight binding representation [5]. Namely it acts on the lattice $\mathbb{Z}^{2}$ as a function of the so-called "magnetic translations" [25] $U$ and $V$. These are two unitaries submitted to the following commutation rule:

$$
U V=e^{2 \mathbf{i} \pi \alpha} V U,
$$

where $\alpha=\phi / \phi_{0}$ is the ratio between the flux $\phi$ in the unit cell of the lattice and the flux quantum $\phi_{0}=e / h$.

The abstract $C^{*}$-algebra $\mathscr{t}_{\alpha}$ generated by two such unitaries was introduced by Rieffel [22] and subsequently Connes [9] showed that it has a differential structure which makes it a non-commutative smooth manifold. Moreover, given an interval $I$ in the real line, the field $\alpha \in I \mapsto A_{\alpha}$ is a continuous field of $C^{*}$-algebras $[10,23]$ and we will denote by $\mathscr{C}_{I}$ the $C^{*}$-algebra it generates.

One of the most famous examples of Hamiltonian built in this way is the so-called "Harper model" [12] given by:

$$
H_{\text {Harper }}=U+U^{*}+V+V^{*},
$$

* URA 505, CNRS

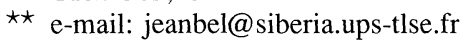


the spectrum of which having been studied by a large number of authors [4] but it was not until 1976 that the work of Hofstadter [15] revealed its fractal nature as the magnetic field changes.

Actually many properties of Harper's model are shared by a large class of self adjoint elements of $A_{I}$. In particular, their gap boundaries are continuous functions of $\alpha$ [11]. Moreover, a semiclassical analysis [5, 13, 20] shows that for a generic set of smooth self adjoint elements $H \in \mathscr{A}_{I}$ the gap boundaries have a discontinuous derivative at each rational value of $\alpha$.

The regularity properties of the gap boundaries for the Harper model have been investigated recently by several authors. First, Choi, Elliot and Yui [8] proved that they are Hölder continuous with exponent 1/3. This result was actually improved by Avron, van Mouche and Simon [2] who got an exponent 1/2. In view of the semiclassical analysis, this last result is optimal without further restrictions. For indeed, whenever a gap closes at say $\alpha=p / q$, its width vanishes like $O(\sqrt{|\alpha-p / q|})$ if $\alpha$ is near $p / q$ $[13,20]$. However we may expect a better result for $\alpha$ 's for which the gap under study does not close.

Our main results in the present work concern not only Harper's model, but a large class of smooth Hamiltonians in $\mathscr{b}_{I}$ as well. This is of interest in view of the construction of effective Hamiltonians by means of the so-called "Peierls substitution" $[18,5,14]$, for which Harper's model is only a rough approximation. Our first result is that the spectrum edges of a self adjoint $\mathscr{C}^{1,3+\varepsilon}$ element in $\mathscr{A}_{I}$ are Lipshitz continuous functions of $\alpha$. The class $\mathscr{C}^{1,3+\varepsilon}$ is defined in Sect. 2. As a corollary, the gap boundaries of an open gap of such an operator are also Lipshitz continuous, but the Lipshitz constant diverges near the values of $\alpha$ for which the gap closes.

Most results previously known in this field of research concern Harper's model. Various techniques have been used: Schrödinger operator techniques, transfer matrix methods, pseudodifferential calculus. We believe however that non-commutative geometry constitutes a natural framework to study extensions to other realistic models, such as the ones one gets with effective single-band Hamiltonians representing the motion of a crystal-two-dimensional electron in a uniform magnetic field [5, 14]. Such models have been however studied by physicists [3, 4, 20, 24].

Because many results we need are scattered in the literature, we have preferred a self-contained exposition of the material. The paper is organized as follows. In Sect. 2 we describe the technical background and we give the precise statements of our results. Section 3 concerns a general proof of Elliot's theorem [11] on the continuity of gap edges. This part is not new but it is included for completeness. In Sect. 4 we prove the first part of our result, namely the Lipshitz continuity of the gap edges once we admit the Lipshitz continuity of the spectral edges. Section 5 is devoted to giving some more technical tools in Weyl's calculus, whereas the proof of the main theorem is given in Sect. 6.

Finally we would like to point out that a semiclassical analysis makes it possible to show that the gap edges are right and left differentiable at each rational value of $\alpha$. The "Wilkinson-Rammal" formula, proved in [24, 19, 13] for Harper's equation and extended to the case of arbitrary elements in some of the $\mathscr{C}^{l, n}[5,20]$, gives the value of these derivatives. In particular, it shows that the gap edges have a discontinuity of their first derivative at each rational point, at least generically in $\mathscr{C}^{l, n}$. So we cannot get a better estimate. We suspect however that these gap edges are actually $\mathscr{C}^{\infty}$ at each irrational $\alpha$ whenever the Hamiltonian belongs to $\mathscr{C} \infty, \infty$. 


\section{The Main Results}

Let $I$ be some closed interval contained in the real line. For $k \in \mathbb{N}$, the algebra of smooth polynomials $\mathscr{P}_{I}^{k}$ is the complex vector space of finite sums

$$
A=\sum_{m \in \mathbb{Z}^{2}} a_{m} W_{I}(m),
$$

where the $a_{m}$ 's are elements of $\mathscr{C}^{k}(I)$, with all but a finite number of them vanishing, and the $W_{I}(m)$ 's are abstract unitaries submitted to the following relations:

(i) every $a \in \mathscr{C}^{k}(I)$ commutes with $W_{I}(m)$,

(ii) $W_{I}(m) W_{I}\left(m^{\prime}\right)=W_{I}\left(m+m^{\prime}\right) \lambda^{-m \wedge m^{\prime}}$, where $m \wedge m^{\prime}=m_{1} m_{2}^{\prime}-m_{2} m_{1}^{\prime}$, and $\lambda$ is the map $\alpha \in I \mapsto e^{\mathbf{i} \pi \alpha} \in \mathbb{C}$,

(iii) $W_{I}(m)^{*}=W_{I}(m)^{-1}=W_{I}(-m)$.

Then $\mathscr{P}_{I}^{k}$ becomes a ${ }^{*}$-algebra. Let $\operatorname{Rep}\left(\mathscr{P}_{I}^{k}\right)$ denote the set of unitary equivalence classes of representations of $\mathscr{P}_{I}^{k}$ on separable Hilbert spaces. A norm can be defined on $\mathscr{P}_{I}^{k}$ by:

$$
\|A\|_{I}=\sup _{\pi \in \operatorname{Rep}\left(\mathscr{F}_{I}^{k}\right)}\|\pi(A)\|
$$

The algebra $\mathscr{b}_{I}$ is the completion of $\mathscr{P}_{I}^{k}$ under this norm. It is a $C^{*}$-algebra with unit.

Given $J \subset I$ a subinterval, we denote by $\varrho_{I, J}$ the restriction map defined on $A \in \mathscr{P}_{I}^{k}$ (cf. (3)) by:

$$
\varrho_{I, J}(A)=\left.\sum_{m \in \mathbb{Z}^{2}} a_{m}\right|_{J} W_{J}(m) .
$$

Then $\varrho_{I, J}$ extends as a ${ }^{*}$-homomorphism from $\mathscr{A}_{I}$ into $\mathscr{\ell}_{J}$. For $J=\{\alpha\}$ we will use the notation $\mathscr{\ell}_{\alpha}$ and $\varrho_{\alpha}$ instead of $\mathscr{\ell}_{\{\alpha\}}$ and $\varrho_{I,\{\alpha\}}$. One can then show that [4]:

$$
\|A\|_{I}=\sup _{\alpha \in I}\left\|\varrho_{\alpha}(A)\right\| \text {. }
$$

The map $\tau_{I, \alpha}: A \in \mathscr{P}_{I}^{k} \mapsto a_{0}(\alpha) \in \mathbb{C}$ defines a normalized positive trace on $\mathscr{b}_{I}$. Moreover, for any $A \in \mathscr{A}_{I}$, the map $\tau_{I}(A): \alpha \in I \mapsto \tau_{I, \alpha}(A)$ is continuous and $\tau_{I}$ then defines a positive linear map from $\mathscr{A}_{I}$ into $\mathscr{C}(I)$. We remark that $\tau_{\{\alpha\}, \alpha}=\tau_{I, \alpha} \circ \varrho_{\alpha}$. To shorten notation, we will write $\tau_{\alpha}$ for $\tau_{I, \alpha}$ unless some confusion arises.

We define an action of $\mathbb{T}^{2}$ on $\mathscr{A}_{I}$ by ${ }^{*}$-automorphisms by setting:

$$
\eta_{k}^{I}\left(W_{I}(m)\right)=e^{\mathbf{i} k m} W_{I}(m),
$$

where $k=\left(k_{1}, k_{2}\right) \in \mathbb{T}^{2}, m=\left(m_{1}, m_{2}\right) \in \mathbb{Z}^{2}$ and $k m=k_{1} m_{1}+k_{2} m_{2}$. This group is pointwise-norm-continuous. Its infinitesimal generators are the two ${ }^{*}$-derivations defined by:

$$
\partial_{\mu} A=\left.\frac{\partial}{\partial k_{\mu}} \eta_{k}^{I}(A)\right|_{k=0} .
$$

We also define on $\mathscr{P}_{I}^{1}$ a linear map $\delta$ called the "Itô derivative" [5] by:

$$
\delta A=\sum_{m \in \mathbb{Z}^{2}} \frac{\partial a_{m}}{\partial \alpha} W_{I}(m) .
$$


It satisfies the following properties:

(i) $\delta\left(A^{*}\right)=(\delta A)^{*}$,

(ii) $\delta(A B)=\delta A B+A \delta B-\mathbf{i} \pi\left(\partial_{1} A \partial_{2} B-\partial_{2} A \partial_{1} B\right)$.

In the following if $r=\left(r_{1}, r_{2}\right) \in \mathbb{N}^{2}$ we adopt the notations $|r|=\left|r_{1}\right|+\left|r_{2}\right|$, $r !=r_{1} ! r_{2}$ ! and $\partial^{r}=\partial_{1}^{r_{1}} \partial_{2}^{r_{2}}$. Moreover, whenever $\nu$ is a real positive number smaller than one, we set

$$
\partial_{\mu}^{\nu} A=\frac{\Gamma(\nu) \sin (\pi \nu)}{\pi} \int_{0}^{\infty} \frac{d t}{t^{\nu+1}}\left(2 A-\eta_{t e_{\mu}}^{I}(A)-\eta_{-t e_{\mu}}^{I}(A)\right),
$$

whenever the integral exists in norm, and where $e_{1}, e_{2}$ are the canonical basis in $\mathbb{R}^{2}$. If $l$ is an integer, and $n$ is a non-negative real number, the space $\mathscr{C}^{l, n}\left(\mathscr{b}_{I}\right)$ is the set of elements of $\mathscr{A}_{I}$ such that $\left\|\delta^{s} \partial^{r}(A)\right\|$ is finite for $0 \leq s \leq l, 0 \leq 2 s+|r| \leq n$. It can be endowed with a norm which makes it a Banach ${ }^{*}$-algebra (see Lemma 3 below). It is obviously dense in $\mathscr{C}_{I}$.

We also introduce a Sobolev norm on $\mathscr{P}_{I}^{\infty}$ in the following way whenever $l$ is an integer, $n$ is any non-negative real number and $\Delta=-\partial_{1}^{2}-\partial_{2}^{2}$ denotes the Laplace operator acting on $\mathscr{t}_{I}$ :

$$
\|A\|_{\not{H} l, n}=\sup _{\alpha \in I}\left\{\sup _{s \leq l, s+2 r \leq n} \tau_{\alpha}\left(\left|\delta^{s} \Delta^{r}(A)\right|^{2}\right\}^{1 / 2} .\right.
$$

The completion of $\mathscr{P}_{I}^{\infty}$ under this norm will be the space $\mathscr{H}^{l, n}\left(\mathscr{A}_{I}\right)$.

In the special case $l=n=0, I=\{\alpha\}$, this space will be denoted $\mathscr{L}^{2}\left(\mathscr{C}_{\alpha}\right)$. It is then a Hilbert space endowed with the inner product $\langle A \mid B\rangle_{\alpha}=\tau_{\alpha}\left(A^{*} B\right)$. It is easy to check that the $W_{\alpha}(m)$ 's give an orthonormal basis in this space, and thus a natural unitary mapping $\mathscr{F}$, called the "Fourier transform," between $\mathscr{L}^{2}\left(\mathscr{C}_{\alpha}\right)$ and $l^{2}\left(\mathbb{Z}^{2}\right)$. In particular we get for $A, B$ as in (3):

$$
\tau_{\alpha}\left(A^{*} B\right)=\sum_{m \in \mathbb{Z}^{2}} \overline{a_{m}} b_{m} .
$$

Actually this space is the space of the GNS representation [17] for the trace, the GNS representation $\pi_{\alpha}$ being given by $\pi_{\alpha}(A) B=\varrho_{\alpha}(A) \widehat{\varrho}_{\alpha}(B), A, B \in \mathscr{b}_{I}$, where $\widehat{\varrho}_{\alpha}$ is the canonical imbedding of $\mathscr{t}_{I}$ into $\mathscr{L}^{2}\left(\mathscr{C}_{\alpha}\right)$. By Fourier transform we check that:

$$
\pi_{\alpha}\left(W_{I}\left(m_{0}\right)\right) \psi(m)=e^{\mathbf{i} \pi \alpha m_{0} \wedge m} \psi\left(m-m_{0}\right), \quad \psi \in l^{2}\left(\mathbb{Z}^{2}\right) .
$$

The previous formula shows that the Hilbert space of the GNS representation can be taken independently of $\alpha$ and that, moreover, the map $\alpha \in I \mapsto \pi_{\alpha}(A) \in \mathscr{B}\left(l^{2}\left(\mathbb{Z}^{2}\right)\right)$ is strongly continuous for any $A \in \mathscr{A}_{I}$. Using the Fourier transform, we can check that $\pi_{\alpha}$ is faithful on $\mathscr{C}_{\alpha}$ namely, that $\left\|\pi_{\alpha}(A)\right\|=\left\|\varrho_{\alpha}(A)\right\|$ for any $A \in \mathscr{A}_{I}$.

The first result we will prove here is actually due to Elliot [11].

Theorem 1. Let $H=H^{*}$ be a selfadjoint element of $\mathscr{A}_{I}$. Then the gap edges of the spectrum of $\varrho_{\alpha}(H)$ are continuous functions of $\alpha$.

This result tells us in particular, that the spectrum is a continuous function of the magnetic field. It has actually been proved for Schrödinger operators in a magnetic field by Nenciu [16] and Avron and Simon [1]. But the $C^{*}$-algebra proof that we will provide below, for the sake of completeness of this paper, works as well for a much larger class of pseudodifferential operators. It has been used also to prove some continuity property of the spectrum of Hamiltonians on quasicrystals [6]. 
Our main new results in this paper are summarized in the following two theorems:

Theorem 2. Given $\varepsilon>0$ let $H=H^{*}$ be a selfadjoint element of $\mathscr{H}^{1,3+\varepsilon}\left(\mathscr{A}_{I}\right)$. Let $E_{+}(\alpha), E_{-}(\alpha)$ denote respectively the supremum and the infimum of the spectrum of $\varrho_{\alpha}(H)$. Then $E_{+}$and $E_{-}$are Lipshitz continuous functions, namely, there is a positive constant $C(\varepsilon)$ such that:

$$
\left|E_{ \pm}(\alpha)-E_{ \pm}\left(\alpha^{\prime}\right)\right| \leq C(\varepsilon)\|H\|_{\mathscr{H} 1,3+\varepsilon}\left|\alpha-\alpha^{\prime}\right|
$$

Let $H$ be as in Theorem 2. Then a gap $g$ of $H$ is, for each $\alpha \in I$, a bounded connected component $g(\alpha)$ of the complement of the spectrum of $\varrho_{\alpha}(H)$, such that $\cup_{\alpha \in I} g(\alpha) \times\{\alpha\}$ is a connected component of the complement of $\underset{\alpha \in I}{\cup} \operatorname{Sp}\left(\varrho_{\alpha}(H)\right) \times\{\alpha\}$ in $\mathbb{R} \times I$. It is thus a family of (possibly empty) open intervals of the form $\left(E_{-}^{g}(\alpha), E_{+}^{g}(\alpha)\right)$. Its width will be denoted by $\mathscr{W}^{g}(\alpha)=E_{+}^{g}(\alpha)-E_{-}^{g}(\alpha)$.

Our second result is then:

Theorem 3. Let $H=H^{*}$ be a selfadjoint element of $C^{1,3+\varepsilon}\left(\mathscr{\ell}_{I}\right)$. Let $g$ be a gap. Then the gap boundaries $E_{ \pm}^{g}$ are Lipshitz continuous functions at each $\alpha$ for which the gap width is positive; namely:

$$
\left|E_{ \pm}^{g}(\alpha)-E_{ \pm}^{g}\left(\alpha^{\prime}\right)\right|<C(H)\left\{\sup _{\alpha \leq \beta \leq \alpha^{\prime}} \mathscr{W}^{g}(\beta)\right\}^{-5}\left|\alpha-\alpha^{\prime}\right|
$$

where $C(H)$ is a constant which only depends upon the norm of $H$ in $C^{1,3+\varepsilon}\left(A_{I}\right)$.

Theorem 2 may probably be improved by more refined estimates to get the norm $\|H\|_{1,2+\varepsilon}$ instead of the Sobolev norm. It should also be possible to improve the estimate in Theorem 3 which is certainly not optimal for it does not give the Hölder exponent $1 / 2$ when the gap closes. However an optimal bound should rather be of the same form with the exponent -5 replaced by -1 .

\section{The Elliott-Continuity Theorem}

The aim of this section is to prove the Elliott continuity Theorem 1 . In order to proceed, let us introduce the following definition. Let $K=(K(\alpha))_{\alpha \in I}$ be a family of compact subsets of the complex plane. We will say that $K$ is "outer continuous at $\alpha_{0} \in I^{\prime \prime}$ whenever given any closed subset $F$ such that $K\left(\alpha_{0}\right) \cap F=\emptyset$, there is $\delta>0$ such that if $\left|\alpha-\alpha_{0}\right| \leq \delta$ then $K(\alpha) \cap F=\emptyset$. In much the same way we will say that $K$ is "inner continuous at $\alpha_{0} \in I$ " whenever given any open set $O$ such that $K\left(\alpha_{0}\right) \cap O \neq \emptyset$, there is $\delta>0$ such that if $\left|\alpha-\alpha_{0}\right| \leq \delta$ then $K(\alpha) \cap O \neq \emptyset$. If $K$ is both inner and outer continuous at every $\alpha \in I$, we will say that $K$ is continuous on $I$. It is elementary to check that whenever $K$ is a family of compact subsets of the real line, its continuity is equivalent to the continuity of its gap edges.

Then Theorem 1 is a simple corollary of the following more general result:

Proposition 1. Let $A$ be a normal element of $\mathscr{b}_{I}$, namely $A A^{*}=A^{*} A$. Then the spectrum of $A$, namely the family $\operatorname{Sp}_{I}(A)=\left(\operatorname{Sp}\left(\varrho_{\alpha}(A)\right)\right)_{\alpha \in I}$ is continuous on $I$.

The proof of Proposition 1 requires several steps. The first one is provided by the following due to G. Elliott [11], for which we give a slightly different proof based upon the use of the automorphism group $\eta^{I}$ defined in (Eq. 6), [6]. 
Lemma 1. Let $A \in \mathscr{A}_{I}$ be such that $\varrho_{\alpha_{0}}(A)=0$ for some $\alpha_{0} \in I$. Then for every $\varepsilon>0$, there is $\delta>0$ such that if $\left|\alpha-\alpha_{0}\right| \leq \delta$ then $\left\|\varrho_{\alpha}(A)\right\| \leq \varepsilon$.

Proof. Let us consider first an element $A \in \mathscr{P}_{I}^{0}$ given as in (Eq. 3). Then $\varrho_{\alpha_{0}}(A)=0$ implies $a_{m}\left(\alpha_{0}\right)=0$ for every $m \in \mathbb{Z}^{2}$. Since the $a_{m}$ 's are continuous on $I$, and since only a finite number $N$ of them do not vanish, there is certainly some $\delta>0$ such that if $\left|\alpha-\alpha_{0}\right| \leq \delta$, then $\left|a_{m}(\alpha)-a_{m}\left(\alpha_{0}\right)\right| \leq \varepsilon / N$. Thus $\left\|\varrho_{\alpha}(A)\right\| \leq \sum_{m}\left|a_{m}(\alpha)\right| \leq \varepsilon$.

Now, let $A \in \mathscr{b}_{I}$ and let us consider $\eta_{k}^{I}(A)$ for $k \in \mathbb{T}^{2}$. By construction,

$$
\varrho_{\alpha_{0}} \circ \eta_{k}^{I}(A)=\eta_{k}^{\alpha} \circ \varrho_{\alpha_{0}}(A)=0 .
$$

If now $f \in \mathscr{C}\left(\mathbb{T}^{2}\right)$ we set:

$$
\eta_{f}^{I}(A)=\int_{k \in \mathbb{T}^{2}} \frac{d^{2} k}{4 \pi^{2}} f(k) \eta_{k}^{I}(A)
$$

Then $\varrho_{\alpha_{0}} \circ \eta_{f}^{I}(A)=0$. Let us now choose a sequence $f_{n}$ in $\mathscr{C}^{\omega}\left(\mathbb{T}^{2}\right)$ having the following properties:

i) for all $n \in \mathbb{N}, f_{n}$ is a trigonometric polynomial;

(ii) $f_{n}$ is a non-negative function on $\mathbb{T}^{2}$;

(iii) $\int_{n}^{n} f_{n}(k) d^{2} k / 4 \pi^{2}=1$;

(iv) for every $\delta>0$,

$$
\lim _{n \mapsto \infty} \int_{|k|>\delta} \frac{d^{2} k}{4 \pi^{2}} f_{n}(k)=0 .
$$

An example of such a sequence is provided by the Fejer polynomials $F_{N}$ :

$$
F_{N}(k)=\frac{\sin ^{2}\left((2 N+1) k_{1} / 2\right) \sin ^{2}\left((2 N+1) k_{2} / 2\right)}{(2 N+1)^{2} \sin ^{2}\left(k_{1} / 2\right) \sin ^{2}\left(k_{2} / 2\right)} .
$$

We set $A_{n}=\eta_{f_{n}}^{I}(A)$. Suppose for a moment that $A$ is in $\mathscr{P}_{I}^{0}$ and given by (Eq. $3)$. Then the coefficients of $A_{n}$ are given by $a_{m}^{n}=a_{m} \tilde{f}_{n}(m)$ with $\tilde{f}_{n}(m)$ being the $m^{\text {th }}$ Fourier coefficient of the polynomial $f_{n}$. In particular the coefficients of $A_{n}$ vanish outside the support of $\tilde{f}_{n}$. Moreover, $\lim _{n \mapsto \infty}\left\|A_{n}-A\right\|_{I}=0$. By a $3 \varepsilon$-argument the same will be true for $A_{n}$ whenever $A \in \mathscr{b}_{I}$ and therefore $A_{n} \in \mathscr{P}_{I}^{0}$, and also $\lim _{n \mapsto \infty}\left\|A_{n}-A\right\|_{I}=0$ will still be satisfied. Thus in this way, we get a controlled approximation of $A$ by trigonometric polynomials.

Thus, given $\varepsilon>0$, there exists $N \in \mathbb{N}$ such that if $n \geq N,\left\|A-A_{n}\right\|_{I} \leq \varepsilon / 2$. Choosing now such $n$, we get:

$$
\left\|\varrho_{\alpha}(A)\right\| \leq\left\|\varrho_{\alpha}\left(A-A_{n}\right)\right\|+\left\|\varrho_{\alpha}\left(A_{n}\right)\right\| \leq \varepsilon / 2+\left\|\varrho_{\alpha}\left(A_{n}\right)\right\| .
$$

At last, we choose $\delta>0$ such that $\left|\alpha-\alpha_{0}\right| \leq \delta \Rightarrow\left\|\varrho_{\alpha}\left(A_{n}\right)\right\| \leq \varepsilon / 2$. Then we have for such $\alpha$ 's $\left\|\varrho_{\alpha}(A)\right\| \leq \varepsilon$.

As a corollary of the previous Lemma 1 we get:

Lemma 2. If $A$ is normal in $\mathscr{C}_{I}$, then $\operatorname{Sp}_{I}(A)$ is outer continuous. 
Proof. Let $F$ be a closed subset of the complex plane such that $\operatorname{Sp}\left(\varrho_{\alpha_{0}}(A)\right) \cap F=\emptyset$. Then, by Urysohn's lemma [7], there is a continuous function $g$ on the complex plane, vanishing on the spectrum of $\varrho_{\alpha_{0}}$ and equal to 1 on $F$. Since $A$ is normal, the element $g(A)$ is well defined in $\mathscr{\ell}_{I}$, and satisfies $\varrho_{\alpha_{0}}(g(A))=0$. By the Lemma 1 , there is $\delta>0$ such that $\sup _{z \in \operatorname{Sp}\left(\varrho_{\alpha}(A)\right)}|g(z)|=\left\|\varrho_{\alpha}(g(A))\right\| \leq 1 / 2$ which implies that $\operatorname{Sp}\left(\varrho_{\alpha}(A)\right) \cap F=\emptyset$.

Proof of Theorem 1(end). Let $A$ be a normal element of $A_{I}$. Let also $O$ be an open set in the complex plane such that $\operatorname{Sp}\left(\varrho_{\alpha_{0}}(A)\right) \cap O \neq \emptyset$. This is equivalent to saying that $\operatorname{Sp}\left(\pi_{\alpha_{0}}(A)\right) \cap O \neq \emptyset$, where $\pi_{\alpha}$ is the GNS representation acting on $l^{2}\left(\mathbb{Z}^{2}\right)$ defined by (11). Suppose there is some sequence $\alpha_{n}$ converging to $\alpha_{0}$ in $I$, such that $\operatorname{Sp}\left(\pi_{\alpha_{n}}(A)\right) \cap O=\emptyset$ for all $n$ 's. By the strong continuity of the family $\pi_{\alpha}(A)$, this would imply [21] $\operatorname{Sp}\left(\pi_{\alpha_{0}}(A)\right) \cap O=\emptyset$, a contradiction.

\section{Proof of Theorem 3}

This section is devoted to the proof of Theorem 3 assuming that Theorem 2 holds. First of all we will need some technical results.

Lemma 3. The *-algebra $\mathscr{C}^{l, n}\left(\mathscr{l}_{I}\right)$ can be endowed with a family $\|\cdot\|_{l, n, \xi}$ of (equivalent) norms indexed by $\xi \in \mathbb{R}_{+}$having the following properties:

(i) These norms are algebraic, namely $\|A B\|_{l, n, \xi} \leq\|A\|_{l, n, \xi}\|B\|_{l, m, \xi}$, and $\left\|A^{*}\right\|_{l, n, \xi}=\|A\|_{l, n, \xi}$;

(ii) $\lim _{\xi \rightarrow 0}\|A\|_{l, n, \xi}=\|A\|_{I}$.

Proof. These norms are defined by

$$
\|A\|_{l, n, \xi}=\sum_{0 \leq s \leq l, 0 \leq 2 s+|r| \leq 2} \frac{\xi^{2 s+|r|} c(s,|r|)}{s !^{2} r !}\left\|\delta^{s} \partial^{r} A\right\|,
$$

where the constants $0<c(s, t), s, t \in \mathbb{N}$ are constrained by the following inequalities:

$$
\sum_{0 \leq j \leq t, t^{\prime}, l-\left(s+s^{\prime}\right)} \frac{t ! t^{\prime} ! \pi^{3} c\left(s+s^{\prime}+j, t+t^{\prime}-2 j\right)}{j !^{2}(t-j) !\left(t^{\prime}-j\right) !} \leq c(s, t) c\left(s^{\prime}, t^{\prime}\right),
$$

provided $s, s^{\prime} \leq l, 2 s+t \leq n, 2 s^{\prime}+t^{\prime} \leq n$. That such inequalities can be satisfied is due to the finiteness of $l$ and $n$. (Just multiply an arbitrary family of numbers by a sufficiently large number). In these expressions, the sum runs over the integers but for the last terms for which $r$ may have a fractional part. Then, one replaces $r_{\mu}$ in $r$ ! and in $|r|$ by $\widehat{r}_{\mu}$, where $\widehat{n}$ is the smallest integer greater than or equal to $n$. The lemma is then proved by inspection.

Lemma 4. Let $A$ be an element of $\mathscr{C}^{l, n}\left(A_{I}\right)$ invertible in $\mathscr{A}_{I}$. Then $A$ is invertible in $\mathscr{C}^{l, n}\left(A_{I}\right)$.

Proof. The formula $A^{-1}=A^{*}\left(A A^{*}\right)^{-1}$ shows that it suffices to consider the case for which $A$ is positive. Then let $a_{ \pm}$be the infimum and the supremum of the spectrum of $A$ in $b_{I}$ respectively. Then $0<a_{-} \leq a_{+}$. We can also express $A$ in the form:

$$
A=\frac{a_{+}+a_{-}}{2} \mathbf{1}+\frac{a_{+}-a_{-}}{2} B
$$


where $B$ is selfadjoint of norm at most 1 . If $a=\left(a_{+}+a_{-}\right) / 2$ and $\theta=\left(a_{+}-\right.$ $\left.a_{-}\right) /\left(a_{+}+a_{-}\right)$, we get $A^{-1}=a^{-1}(\mathbf{1}+\theta B)^{-1}$. Since $0 \leq \theta\|B\|_{I}<1$, the Neumann series for $A^{-1}$ converges in $\mathscr{A}_{I}$. But if we choose $\xi>0$ small enough to insure that $\theta\|B\|_{l, n, \xi}<1$ (see Lemma 3), this series also converges in $\mathscr{C}^{l, n}\left(\mathscr{C}_{I}\right)$, producing an inverse of $A$ in $\mathscr{C}^{l, n}\left(\mathscr{A}_{I}\right)$.

Lemma 5. Let $A$ be an element in $\mathscr{C}^{l, n}\left(\mathscr{C}_{I}\right)$ invertible in $\mathscr{A}_{I}$. Then there exists a constant $C_{1}(l, n)$ depending only upon $l$ and $n$ such that

$$
\left\|A^{-1}\right\|_{l, n, \xi=1} \leq C_{1}(l, n)\left\|A^{-1}\right\|_{I}^{\widehat{n}+1}\|A\|_{l, n, \xi=1}^{\widehat{n}},
$$

where $\widehat{n}$ is the smallest integer greater than or equal to $n$.

Proof. We use the following two relations valid for $A \in \mathscr{C}^{1,2}\left(\mathscr{C}_{I}\right)$ :

$$
\partial_{\mu}\left(A^{-1}\right)=-A^{-1}\left(\partial_{\mu} A\right) A^{-1}
$$

and

$$
\begin{aligned}
\delta\left(A^{-1}\right)= & -A^{-1}(\delta A) A^{-1}+ \\
& -\mathbf{i} \pi\left(A^{-1} \partial_{1} A A^{-1} \partial_{2} A A^{-1}-A^{-1} \partial_{2} A A^{-1} \partial_{1} A A^{-1}\right) .
\end{aligned}
$$

They both come from differentiating the product $A A^{-1}=\mathbf{1}$. If we iterate these formulæ, we can check that $\delta^{s} \partial^{r}\left(A^{-1}\right)$ can be decomposed into a sum of products containing $A^{-1}$ at most $2 s+|r|+1$ times, and terms of the form $\delta^{s_{i}} \partial^{r_{i}}(A)$, where the exponents satisfy $\sum s_{\imath} \leq s$ and $\sum 2 s_{\imath}+\left|r_{i}\right|=2 s+|r|$. Thus if $r$ is a pair of integers, the lemma is proved. If now $0<\nu<1$, the formula (9) applied to $A^{-1}$ shows that one gets an extra power of $A^{-1}$, giving the $\widehat{n}$ instead. The constant $C_{1}(l, n)$ comes from counting all such terms.

Proof of Theorem 3. Let $H=H^{*}$ be in $\mathscr{C}^{l, n}\left(A_{I}\right)$ for some $l \geq 1$ and $n>3$. Let some $\alpha$ be in $I$, and let $g$ be some gap of $H$ opened at $\alpha$. By the Elliott Theorem 1 , this gap does not close in a suitable neighbourhood of $\alpha$. So that, without loss of generality, we choose $I$ small enough to forbid this gap to close on $I$.

We then define $\Sigma_{ \pm}(\alpha)$ to be the convex hull of $\operatorname{Sp}\left(\varrho_{\alpha}(H)\right) \cap\left[E_{+}^{g}(\alpha),+\infty\right)$ and $\operatorname{Sp}\left(\varrho_{\alpha}(H)\right) \cap\left(-\infty, E_{-}^{g}(\alpha)\right]$ respectively. Our assumption implies that these are two disjoint intervals such that the gap between them is precisely the interval $g(\alpha)$ under consideration.

Then let $\Gamma(\alpha)$ be a continuous family of Jordan curves homotopic to the unit circle, oriented anticlockwise, surrounding $\Sigma_{-}(\alpha)$ and not $\Sigma_{+}(\alpha)$. Then we set:

$$
P_{g}=\oint_{\Gamma} \frac{d z}{2 \mathbf{i} \pi} \frac{1}{z-H}, \quad H_{g}=\oint_{\Gamma} \frac{d z}{2 \mathbf{i} \pi} \frac{H}{z-H} .
$$

$P_{g}$ is the eigenprojection of $H$ onto the part of the spectrum contained in $\Sigma_{-}$and is therefore an element of $\mathscr{C}_{I}$. Actually, since $z-H$ is invertible for all $z \in \Gamma$, Lemma 4 implies that it is also invertible in $\mathscr{C}^{l, n}\left(\mathscr{\ell}_{I}\right)$, and therefore both $P_{g}$ and $H_{g}$ belong to $\mathscr{C}^{l, n}\left(\mathscr{b}_{I}\right)$. In particular $H_{g} \in \mathscr{H}^{l, n}\left(A_{I}\right)$. Note that $E_{-}^{g}(\alpha)=\sup \operatorname{Sp}\left(\varrho_{\alpha}\left(H_{g}\right)\right)$. Hence, if $l \geq 1$ and $n>3$, we can apply Theorem 2 to $H_{g}$, and conclude that $E_{-}^{g}$ is a Lipshitz continuous function in $I$, with a Lipshitz constant given by $C\left\|H_{g}\right\|_{. \not H l, n} \leq C^{\prime}\left\|H_{g}\right\|_{l, n}$ 
(where we have omitted the value of $\xi$ which we take here equal to 1 ) with a new numerical constant $C^{\prime}$. Using now Lemma 5 above, we get:

$$
\left\|H_{g}\right\|_{l, n} \leq C_{2}(l, n) \operatorname{Length}(\Gamma)\|H\|_{l, n}^{\widehat{n}+1} \sup _{z \in \Gamma}\left\|\frac{1}{z-H}\right\|_{I}^{\widehat{n}+1} .
$$

Choosing $\Gamma$ optimally, we can bound the norm of the resolvent of $H$ along the curve $\Gamma$ by the inverse of the width of $g$, leading to Theorem 3 , if one chooses $3<n \leq 4$.

\section{Weyl's Calculus}

The proof of Theorem 2 requires some technical preparation. This section is devoted to the part which requires Weyl's calculus. This part is certainly well-known, but we include it here to fix notation and conventions on normalization.

We will consider the Hilbert space $\mathscr{L}^{2}(\mathbb{R})$, and the two selfadjoint operators $K_{1}$, $K_{2}$ defined by $-\mathbf{i} d / d s$ and multiplication by $s$ respectively on the common core given by the Schwarz space $\mathscr{S}(\mathbb{R})$ of rapidly decreasing $\mathscr{C}^{\infty}$ functions on $\mathbb{R}$. We use this notation because of the relation of this problem with Solid State Physics. Moreover we want to restore the symmetry of the phase space between position and momentum. Then we have $\left[K_{1}, K_{2}\right]=-\mathbf{i}$. Weyl's operators are given by $e^{\mathbf{i} K x}$ for $x=\left(x_{1}, x_{2}\right) \in \mathbb{R}^{2}$, where $K x=K_{1} x_{1}+K_{2} x_{2}$. We will also use the notation $K \wedge x=K_{1} x_{2}-K_{2} x_{1}$. The Weyl operators are unitary and satisfy the canonical commutation relation (CCR), namely:

$$
e^{\mathbf{i} K x} e^{\mathbf{i} K y}=e^{\mathbf{i} K(x+y)+\mathbf{i} x \wedge y / 2}, \quad x, y \in \mathbb{R}^{2} .
$$

Actually, $e^{\mathrm{i} K \wedge x}$ represents nothing but the translation by $x$ in phase space, namely, the map

$$
e^{\mathbf{i} K \wedge x} K e^{-\mathbf{i} K \wedge x}=K+x .
$$

We now consider the groundstate $|0\rangle$ of the "harmonic oscillator" $K_{1}^{2}+K_{2}^{2}$, namely the function $f_{0}(s)=\pi^{-1 / 4} e^{-s^{2} / 2}$. It is well-known fact that this state saturates the Heisenberg inequality. So that in a certain sense, it represents the point $x=0$ in the quantal phase space. To move it around, we then use the Weyl operators, and we set:

$$
T(x)=e^{\mathbf{i} K \wedge x}|0\rangle\langle 0| e^{-\mathbf{i} K \wedge x}, \quad x \in \mathbb{R}^{2} .
$$

Clearly $T(x)$ is a one-dimensional projection. We let now $F$ be a locally integrable function on $\mathbb{R}^{2}$ and let $T(F)$ be the following operator, defined as a weak integral:

$$
T(F)=\int_{\mathbb{R}^{2}} \frac{d^{2} x}{2 \pi} F(x) T(x),
$$

whenever it converges. The following properties hold:

Proposition 2. (i) Positivity: if $F$ is non-negative almost everywhere (with respect to Lebesgue's measure), $T(F)$ is a positive operator.

(ii) Normalization: $T(\mathbf{1})=\mathbf{1}$.

(iii) Boundedness: if $F \in \mathscr{S}^{\infty}\left(\mathbb{R}^{2}\right)$, then $T(F)$ is bounded operator. In addition one has $\|T(F)\| \leq\|F\|_{L^{\infty}}$.

(iv) Trace property: if $F \in \mathscr{L}^{1}\left(\mathbb{R}^{2}\right)$, then $T(F)$ is trace class. One gets actually, $\operatorname{Tr}(|T(F)|) \leq\|F\|_{L^{1}} / 2 \pi$, and also $\operatorname{Tr}(T(F))=\int F(x) d^{2} x / 2 \pi$. 
(v) Hilbert-Schmidt property: if $F \in \mathscr{L}^{2}\left(\mathbb{R}^{2}\right)$, then $T(F)$ is Hilbert-Schmidt. Moreover, one gets $\operatorname{Tr}\left(|T(F)|^{2}\right)^{1 / 2} \leq\|F\|_{L^{2}} / 2 \pi$.

(vi) Weyl's operators: we get the following formula, where the integral converges in the weak sense:

$$
e^{\mathbf{i} K x}=\int_{\mathbb{R}^{2}} \frac{d^{2} y}{2 \pi} e^{x^{2} / 2-\mathbf{i} y x} T(y) .
$$

Proof. (i) is obvious from the definition (20), provided the integral converges. (ii) is a consequence of (vi). (iii) follows immediately from (i) and (ii). Let us prove (iv). Given any pair $\left(e_{n}\right)_{n \geq 0}$ and $\left(f_{n}\right)_{n \geq 0}$ of orthonormal basis in $\mathscr{L}^{2}(\mathbb{R})$, we get, for any $N \in \mathbb{N}$ :

$$
\left|\sum_{n=0}^{N}\left\langle e_{n} \mid T(F) f_{n}\right\rangle\right| \leq\left|\int_{\mathbb{R}^{2}} \frac{d^{2} x}{2 \pi} F(x) \sum_{n=0}^{N}\left\langle e_{n} \mid T(x) f_{n}\right\rangle\right| .
$$

The sum inside the integral of the right-hand-side can be estimated by using a Schwartz inequality, namely:

$$
\left|\sum_{n=0}^{N}\left\langle e_{n} \mid T(x) f_{n}\right\rangle\right| \leq \operatorname{Tr}(T(x))=1 .
$$

If $U$ is the partial isometry entering into the polar decomposition of $T(F)$, and if we choose $f_{n}=U^{*} e_{n}$, the left-hand-side gives $\operatorname{Tr}(|T(F)|)$ in the limit $N \mapsto \infty$, leading to (iv). (v) is then a consequence of (iii) and (iv) via the Riesz-Thorin interpolation theorem [21].

Let us turn now to the formula (21). Let $f, g \in \mathscr{S}(\mathbb{R})$. It is easy to check that:

$$
\left\langle f \mid e^{\mathbf{i} K x} g\right\rangle=\int_{\mathbb{R}} d s \overline{f(s)} g\left(s+x_{1}\right) e^{\mathbf{i} s x_{2}+\mathbf{i} x_{1} x_{2}} .
$$

In particular we get:

$$
\langle f \mid T(y) g\rangle=\int_{-\infty}^{+\infty} d s \int_{-\infty}^{+\infty} d t \overline{f\left(s-y_{2}\right)} g\left(t-y_{2}\right) e^{-\left(s^{2}+t^{2}\right) / 2+\mathbf{i} y_{1}(t-s)} / \sqrt{\pi}
$$

Multiplying by $e^{-\mathbf{i} x y}$ and integrating first with respect to $y_{1}$ gives (using the Parseval identity for Fourier transforms):

$$
\int_{-\infty}^{+\infty} d y_{1} e^{-i x y}\langle f \mid T(y) g\rangle=\int_{-\infty}^{+\infty} \frac{d s}{\sqrt{\pi}} \overline{f(s)} g\left(s-x_{1}\right) e^{-\left(\left(s+y_{2}\right)^{2}+\left(s+y_{2}-x_{1}\right)^{2}\right) / 2} e^{-\mathbf{i} x_{2} y_{2}} .
$$

The integration over $y_{2}$ is now easy for it is a gaussian integral and gives the result, once compared with (Eq. 22).

Before ending this section let us give another technical result. Let $\mathscr{C}_{b}^{2}\left(\mathbb{R}^{2}\right)$ be the space of bounded continuous functions of class $\mathscr{C}^{2}$ with all derivatives bounded. Then: 
Lemma 6. Let $F$ belong to $\mathscr{C}_{b}^{2}\left(\mathbb{R}^{2}\right)$. For $h>0$ set

$$
F_{h}(x)=\int_{\mathbb{R}^{2}} \frac{d^{2} y}{2 \pi h} e^{-y^{2} / 2 h} F(y+x) .
$$

Then

$$
\left|F_{h}(x)-F(x)\right| \leq h\left\|D^{2} F\right\|_{\infty},
$$

where $D^{2} F(x)$ is the $2 \times 2$ matrix of second derivatives of $F$ at $x$, and $\left\|D^{2} F\right\|_{\infty}=$ sup $\left\|D^{2} F(x)\right\|$, the norm of a matrix being the operator norm for the euclidean norm in $\mathbb{C}^{2}$.

Proof. The integral over $y$ is actually a probability measure so that:

$$
F_{h}(x)-F(x)=\int_{\mathbb{R}^{2}} \frac{d^{2} y}{2 \pi h} e^{-y^{2} / 2 h}(F(x+y)-F(x)) .
$$

We use a second order Taylor expansion to get

$$
F_{h}(x)-F(x)=\int_{\mathbb{R}^{2}} \frac{d^{2} y}{2 \pi h} e^{-y^{2} / 2 h}\left\{y \nabla F(x)+\int_{0}^{1} d s(1-s)\langle y| D^{2} F(x+s y) y\right\} .
$$

The first term in the right-hand-side vanishes obviously by the parity of the measure, while the second term is easily bounded by $\mathscr{T}\left\|D^{2} F\right\|_{\infty}$, where

$$
\mathscr{T}=\int_{\mathbb{R}^{2}} \frac{d^{2} y}{2 \pi h} \frac{y^{2}}{2} e^{-y^{2} / 2 h}=h .
$$

This finishes the proof.

Corollary 1. With the notations of Lemma 6 we get:

$$
\left\|F_{h}\right\|_{\infty} \geq\|F\|_{\infty}-h\left\|D^{2} F\right\|_{\infty} .
$$

\section{Proof of Theorem 2}

The proof of Theorem 2 requires again several steps. The first one consists in finding a controlled approximation of $H \in \mathscr{H}^{l, n}\left(\mathscr{C}_{I}\right)$ by trigonometric polynomials. This is the content of the first lemma below.

Given $H=H^{*} \in \mathscr{C}_{I}$, we define its Fourier coefficients as:

$$
h_{m}(\alpha)=\tau_{\alpha}\left(W_{I}(m)^{*} H\right) .
$$

By the very construction of the trace, it is easy to check that this is a continuous function of $\alpha$ on $I$. Since $h \in \mathscr{A}_{I}$, it belongs in particular to $\mathscr{L}^{2}\left(\mathscr{C}_{I}\right)$, so that the Parseval formula gives:

$$
\tau_{\alpha}\left(H H^{*}\right)=\sum_{m \in \mathbb{Z}^{2}}\left|h_{m}(\alpha)\right|^{2}
$$


This sum converges uniformly on $I$. In particular we get:

$$
\|H\|_{\mathscr{H}_{l}, n}=\sup _{\alpha \in I} \sup _{s \leq l, 2 s+2 r \leq n} \sum_{m \in \mathbb{Z}^{2}} m^{4 r}\left|\frac{\partial^{s} h_{m}}{\partial \alpha^{s}}(\alpha)\right|^{2} .
$$

This allows us to conclude that whenever $n-2 l>2$, the series

$$
\sum_{m \in \mathbb{Z}^{2}} \frac{\partial^{s} h_{m}}{\partial \alpha^{s}}(\alpha) W_{\alpha}(m)
$$

converges absolutely and uniformly in $I$ in $\mathscr{A}_{I}$ for $s \leq l$. Let us then define $H_{N}$ to be the polynomial of degree at most $N$ such that:

$$
\varrho_{\alpha}\left(H_{N}\right)=\sum_{|m|_{2}<N} h_{m}(\alpha) W_{\alpha}(m)
$$

Then we obtain:

Lemma 7. For any pair $(l, n)$ such that $l \geq 0, n>1$, and any $0 \leq \nu \leq n-1$, there is a positive constant $C_{3}(\nu)$ such that:

$$
\left\|H-H_{N}\right\|_{I} \leq \frac{C_{3}(\nu)}{N^{\nu}}\|H\|_{\mathscr{K} l, n},
$$

Proof. We easily get

$$
\left\|H-H_{N}\right\|_{I} \leq \sup _{\alpha \in I} \sum_{|m|_{2} \geq N}\left|h_{m}(\alpha)\right|
$$

where $|m|_{2}$ is euclidean norm of the vector $m$. Using the Schwarz inequality, for any $r>1 / 2$ we get:

$$
\left\|H-H_{N}\right\|_{I} \leq\left\{\sum_{|m|_{2} \geq N} \frac{1}{m^{4 r}}\right\}^{1 / 2} \sup _{\alpha \in I}\left\{\sum_{|m|_{2} \geq N} m^{4 r}\left|h_{m}(\alpha)\right|^{2}\right\}^{1 / 2} .
$$

This gives the result by taking $\nu=2 r-1>0$ which in turn, implies $n>1$.

The next step in the proof consists in using a representation of the algebra which allows us to compare the elements $\varrho_{\alpha}(H)$ and $\varrho_{\alpha^{\prime}}(H)$. Actually, since they are in different $C^{*}$-algebras they cannot be compared directly in the abstract set-up. We will assume that $\alpha^{\prime}=\alpha+\delta$ with $\delta>0$.

In order to deal with it, we use the fact that $\mathscr{C}_{\alpha+\delta}$ is isomorphic to the subalgebra of the tensor product $\mathscr{A}_{\alpha} \otimes \mathscr{A}_{\delta}$ generated by the elements $W_{\alpha}(m) \otimes W_{\delta}(m)$, for $m \in \mathbb{Z}^{2}$. Moreover, the Weyl representation $\pi_{W}$ of $\mathscr{b}_{\delta}$ acting on $\mathscr{L}^{2}(\mathbb{R})$ is faithful [4]. This representation is defined by:

$$
\pi_{W}\left(W_{\delta}(m)\right)=e^{\mathbf{i} \sqrt{2 \pi \delta} K m} .
$$

Faithfulness implies in particular that the spectrum does not change by taking the representation instead of the algebra. Hence, omitting the symbol $\pi_{W}$, we easily represent $\varrho_{\alpha+\delta}\left(H_{N}\right)=H_{\alpha+\delta, N}$ as the element of the tensor product $\mathscr{C}_{\alpha} \otimes \mathscr{B}\left(\mathscr{L}^{2}(\mathbb{R})\right)$ given by:

$$
\varrho_{\alpha+\delta}\left(H_{N}\right)=H_{\alpha+\delta, N}=\sum_{|m|_{2}<N} h_{m}(\alpha+\delta) W_{\alpha}(m) \otimes e^{\mathrm{i} \sqrt{2 \pi \delta} K m} .
$$


Using [Eq. (21)] we get the following integral representation:

$$
H_{\alpha+\delta, N}=\int_{\mathbb{R}^{2}} \frac{d^{2} x}{2 \pi} H_{\alpha, N}(\delta, \sqrt{2 \pi \delta} x) \otimes T(x),
$$

where $H_{\alpha, N}(\delta, x)$ is the polynomial in $\mathscr{A}_{\alpha}$ given by:

$$
H_{\alpha, N}(\delta, x)=\sum_{|m|_{2}<N} h_{m}(\alpha+\delta) e^{\pi \delta m^{2} / 2} W_{\alpha}(m) e^{\mathbf{i} x m} .
$$

We note in passing that $H_{\alpha, N}(\delta, x)=\eta_{x}^{\alpha}\left(H_{\alpha, N}(\delta, 0)\right)$, so that the spectrum of this polynomial does not depend upon $x$.

We remind the patient reader, who got to this point of our paper, that $E_{+}(\alpha)$ is the supremum of the spectrum of $H_{\alpha}$. In much the same way, we define $E_{+}^{N}(\alpha)$ to be the supremum of the spectrum of $H_{\alpha, N}$ and $E_{+}^{N}(\alpha, \delta)$ to be the supremum of the spectrum of $H_{\alpha, N}(\delta, x)$ (a quantity which therefore does not depend on $x$ ). It is up to the reader to prefer dealing with the infimum of the spectrum instead; the proof will obviously be similar. Our next result is then summarized in the following:

Lemma 8. Let $(l, n)$ be a pair such that $l \geq 0$ and $n>3$. Then there is a positive constant $C_{4}(n)$ depending only upon $n$ such that the following estimate holds:

$$
E_{+}^{N}(\alpha, \delta)-C_{4}(n) \delta e^{\pi \delta N^{2} / 2}\|H\|_{\mathscr{H} l, n} \leq E_{+}^{N}(\alpha+\delta) \leq E_{+}^{N}(\alpha, \delta) .
$$

Proof. The positivity property of the operator $T(x)$ gives the right-hand-side of our inequality. To get the left-hand-side, we choose any $\varepsilon>0$, and then a state $\omega_{\varepsilon}$ on $\mathscr{C}_{\alpha}$ such that $\omega_{\varepsilon}\left(H_{\alpha, N}(\delta, 0)\right) \geq E_{+}^{N}(\alpha, \delta)-\varepsilon$. We also use on $\mathscr{B}\left(\mathscr{L}^{2}(\mathbb{R})\right)$ the states $\Omega_{x}$ defined by $\Omega_{x}(A)=\operatorname{Tr}(T(x / \sqrt{2 \pi \delta}) A)$. Then we get:

$$
\omega_{\varepsilon} \otimes \Omega_{x}\left(H_{\alpha+\delta, N}\right)=\int_{\mathbb{R}^{2}} \frac{d^{2} y}{4 \pi^{2} \delta} F_{\varepsilon}(y) e^{-(y-x)^{2} / 4 \pi \delta},
$$

provided $F_{\varepsilon}(x)=\omega_{\varepsilon}\left(H_{\alpha, N}(\delta, x)\right)$. Using Lemma 6, its corollary, the definition of $\omega_{\varepsilon}$ and taking the supremum of the left-hand-side over $x$ and over $\varepsilon$ gives:

$$
\sup _{x, \varepsilon} \omega_{\varepsilon} \otimes \Omega_{x}\left(H_{\alpha+\delta, N}\right) \geq \sup _{\varepsilon}\left\{E_{+}^{N}(\alpha, \delta)-\varepsilon-2 \pi \delta\left\|D^{2} F_{\varepsilon}\right\|_{\infty}\right\} \text {. }
$$

It is then not difficult to check that, if $3<2 r \leq n$, and for any $\varepsilon$ :

$$
\begin{aligned}
\left\|D^{2} F_{\varepsilon}\right\|_{\infty} & \leq \sum_{|m|_{2}<N} m^{2}\left|h_{m}(\alpha+\delta)\right| e^{\pi \delta m^{2} / 2} \\
& \leq e^{\pi \delta N^{2} / 2}\left\{\sum_{|m|_{2}<N} \frac{1}{\left(1+m^{2}\right)^{2 r-2}}\right\}^{1 / 2}\|H\|_{\mathscr{H}, n} .
\end{aligned}
$$

This inequality, together with the previous one, gives the result.

It then remains to compare $E_{+}^{N}(\alpha, \delta)$ with $E_{+}^{N}(\alpha)$. It can be done by comparing $H_{\alpha, N}(\delta, x)$ with $H_{\alpha, N}$. Since these are both polynomials in the same algebra, there is no difficulty in doing that. Namely, we get: 
Lemma 9. For any pair $(l, n)$ such that $l \geq 1$ and $n>3$, there is a positive constant $C_{5}(n)$ depending only on $n$ such that

$$
\left\|H_{\alpha, N}(\delta, 0)-H_{\alpha, N}\right\| \leq|\delta| e^{\pi \delta N^{2} / 2} C_{5}(n)\|H\|_{\mathscr{H} l, n} .
$$

Proof. If one uses the definition of the two polynomials and a Taylor expansion in $\delta$, we easily get:

$$
\begin{aligned}
& \left\|H_{\alpha, N}(\delta, 0)-H_{\alpha, N}\right\| \\
& \quad \leq \sum_{|m|_{2}<N} \delta \int_{0}^{1} d s\left|\frac{\partial h_{m}}{\partial \alpha}(\alpha+s \delta)+(\pi / 2) m^{2} h_{m}(\alpha+s \delta)\right| e^{s \pi \delta m^{2} / 2} .
\end{aligned}
$$

We then dominate the exponential term by $e^{\pi \delta N^{2} / 2}$ and use again the Schwarz inequality like in the previous lemmas. One can easily check that the constant $C_{5}(n)$ is given by the square root of the sum $\sum_{m}\left(1+m^{2}\right)^{-2 r}$, for some $r>0$ such that $2 r+2 \leq n$. The convergence is then ensured only if $n>3$. We also need $l \geq 1$ because we use a derivative with respect to $\alpha$ in that estimate.

Proof of Theorem 2 (end). To prove Theorem 2, we just have to glue together the inequalities that we have proved previously in this section. Namely:

$$
\begin{aligned}
\left|E_{+}(\alpha)-E_{+}(\alpha+\delta)\right| \leq & \left|E_{+}(\alpha)-E_{+}^{N}(\alpha)\right|+\left|E_{+}(\alpha+\delta)-E_{+}^{N}(\alpha+\delta)\right| \\
& +\left|E_{+}^{N}(\alpha+\delta)-E_{+}^{N}(\alpha, \delta)\right|+\left|E_{+}^{N}(\alpha, \delta)-E_{+}^{N}(\alpha)\right| .
\end{aligned}
$$

The first two terms of the right-hand side are estimated through (Eq. 23), the third term through (Eq. 27) and the last term through (Eq. 28). This gives:

$$
\left|E_{+}(\alpha)-E_{+}(\alpha+\delta)\right| \leq\left\{\frac{2 C_{3}(\nu)}{N^{\nu}}+\left(C_{4}(n)+C_{5}(n)\right) \delta e^{\pi \delta N^{2} / 2}\right\}\|H\|_{\mathscr{H},, n},
$$

provided $l \geq 1, n>3,0 \leq \nu \leq n-1$. This last inequality holds for any $N$, so that we can optimize it by taking say $N=O\left(\delta^{-1 / 2}\right)$ and $\nu=2$. This gives the desired result and ends this proof at last.

Acknowledgements. I thank the Mittag-Leffler Institute of Mathematics in Sweden for financial support and for providing facilities which permitted this paper to be written.

\section{References}

1. Avron, J.E., Simon, B.: Stability of gaps for periodic potentials under variation of a magnetic field. J. Phys. A: Math. Gen. 18, 2199-2205 (1985)

2. Avron, J., van Mouche, P.H.M., Simon, B.: On the measure of the spectrum for the almost Mathieu operator. Commun. Math. Phys. 132, 103-118, (1990). Erratum in Commun. Math. Phys. 139, 215 (1991)

3. Barelli, A.: Approche algébrique de la limite semi-classique: electrons bidimensionnels en champ magnétique et localisation dynamique. Thèse, Univ. Paul Sabatier, Toulouse, Sept. 1992

4. Barelli, A., Bellissard, J., Fleckinger, R.: 2D Bloch electrons in a uniform magnetic field. In preparation 1993

5. Bellissard, J.: $C^{*}$-algebras in solid state Physics. In: "Operator algebras and applications," Vol. II. Evans, D.E., Takesaki, (eds.) Cambridge: University Press, 1988 
6. Bellissard, J., Iochum, B., Testard, D.: Continuity properties of the electronic spectrum of 1D quasicrystals. Commun. Math. Phys. 141, 353-380 (1991)

7. Bourbaki, N.: Topologie générale. Chap. IX, $\S 4, \mathrm{n}^{0}$ 1. Paris: Hermann 1948

8. Choi, M.D., Elliott, G.A., Yui, N.: Gauss polynomials and the rotation algebra. Invent. Math. 99, 225-246 (1990)

9. Connes, A.: A survey of foliation algebras. In "Operator algebras and applications." Proc. Symposia Pure Math., Vol. I, 38, 521-628 (1982)

10. Dixmier, J.: Les $C^{*}$-algèbres et leurs représentations. Paris: Gauthiers-Villars 1969

11. Elliott, G.: Gaps in the spectrum of an almost periodic Schrödinger operator. C.R. Math. Rep. Acad. Sci. Canada 4, 255-259 (1982)

12. Harper, P.G.: Single band motion of conduction electrons in a uniform magnetic field. Proc. Phys. Soc. Lon. A68, 874-892 (1955)

13. Helffer, B., Sjöstrand, J.: Analyse semi-classique pour l'équation de Harper. II. Bull. Soc. Math. France 117, Fasc. 4, Mémoire 40 (1990)

14. Helffer, B., Sjöstrand, B.: Equation de Schrödinger avec champ magnétique et équation de Harper. Springer Lecture Notes in Physics 345 1989, pp. 118-197

15. Hofstadter, D.G.: Energy levels and wave functions of Bloch electrons in rational or irrational magnetic field. Phys. Rev. B14, 2239-2249 (1976)

16. Nenciu, G.: Stability of energy gaps under variation of the magnetic field. Lett. Math. Phys. 11, 127-132, (1986)

17. Pedersen, G.: $C^{*}$-algebras and their automorphism groups. New York: Academic Press 1979

18. Peierls, R.: Zur Theorie des Diamagnetismus von Leitungselektronen. Z. Phys. 80, 763-791, (1933)

19. Rammal, R.: In: Physics and fabrication of microstructures. Kelly, M., Weisbuch, C., eds., Berlin-Heidelberg-New York: Springer 1986, p. 303

20. Rammal, R., Bellissard, J.: An algebraic semiclassical approach to Bloch electrons in a magnetic field. J. Phys. France 51, 1803-1830, (1990)

21. Reed, M., Simon, B.: Methods of modern mathematical physics, Vol. 1, Chap. VIII. New York: Academic Press 1980

22. Rieffel, M.A.: $C^{*}$-algebras associated with the irrational rotation. Pac. J. Math. 95, 415-419, (1981)

23. Tomiyama, J.: Topological representations of $C^{*}$-algebras. Tokohu Math. J. 14, 187-204 (1962)

24. Wilkinson, M.: An example of phase holonomy in WKB theory, J. Phys. A: Math. Gen. 17, 3459-3476 (1984)

25. Zak, J.: Magnetic translation group. Phys. Rev. A134, 1602-1607 (1964); Magnetic translation Group. II. Irreducible representations. Phys. Rev. A134, 1607-1611 (1964)

Communicated by H. Araki 
